\title{
Synthesis of Glycosaminoglycan-Like Copolysaccharide Derivative. The System of Anhydroglucosamine Monomer and Carboxyl-Containing Monomer Has a Tendency to Alternate
}

\author{
Ken-Ichi Kanno, Toshikazu Minamisawa, and Kenichi Hatanaka ${ }^{\dagger}$ \\ Department of Biomolecular Engineering, Tokyo Institute of Technology, \\ 4259 Nagatsuta-cho, Midori-ku, Yokohama 226, Japan
}

(Received February 14, 1997)

KEY WORDS Alternate Copolymer/Ring-Opening Polymerization

/ Polysaccharide / Glycosaminoglycan / Anhydrosugar Monomer /

Glycosaminoglycans (GAGs), in which glucosamine and uronic acid units alternate along the chain, play various important roles in vivo. The relationship between chemical structures and biological activities of GAGs has been revealed by using GAG derivatives which were prepared by the modification reactions such as de- $O$-sulfation, ${ }^{1}$ de- $N$-sulfation, ${ }^{2}$ over-sulfation, ${ }^{3}$ degradation, ${ }^{4}$ and etc. ${ }^{5,6}$ In some cases, biological reactions of artificial polysaccharides or artificial oligosaccharides have revealed the structure-function relationship of the polysaccharides. For example, heparin is well known for its anticoagulant effect mediated by the serine protease inhibitor antithrombin III (ATIII). ${ }^{7}$ Boeckel et al. has synthesized highly-active pentasaccharide sequence of heparin and its analogues, and it was suggested that the flexibility of L-iduronic acid unit in heparin played an important function for binding to ATIII. ${ }^{8}$ Moreover, they designed and synthesized a new analogue of heparin pentasaccharide, having higher activity than heparin pentasaccharide, by introducing a hydrophobic substituent into the carbohydrate. Other investigations about the relationship between the structures and the activities of polysaccharides have been also reported. ${ }^{9-11}$ However, artificial polysaccharides have been rarely used in biological investigations, because strictly designed polysaccharides with high molecular weights are difficult to synthesize. Polysaccharides consisting of disaccharide repeating units, such as GAGs have never been synthesized by polymerization reaction. In this paper, the synthesis of hetero-polysaccharides with various contents of carboxyl and amino groups is reported.

\section{EXPERIMENTAL}

\section{General Methods}

$270-\mathrm{MHz}{ }^{1} \mathrm{H}$ NMR spectra were recorded on a JEOL EX-270 spectrometer in $\mathrm{CDCl}_{3}$ using tetramethylsilane as internal reference. Gel-permeation chromatography was carried out on $1 \%$ solutions of the polymer in tetrahydrofuran with a Shimadzu liquid chromatograph (model LC-9A, columns: GPC-802, 803, and 804). The number-average molecular weights calculated by GPC were based on polystyrene calibration curve.

\footnotetext{
To whom correspondence should be addressed.
}

\section{Monomers}

1,6-Anhydro-3,4-di- $O$-benzyl-2-deoxy-2-phthalimido$\beta$-D-glucopyranose ${ }^{12}$ (1) and 1,6-anhydro-2,4-di- $O$ benzyl-3-O-1-(methoxycarbonyl)ethyl- $\beta$-D-glucopyranose $^{13}$ (2) were synthesized from levoglucosan according to the literatures.

\section{Copolymerization}

Copolymerizations of $\mathbf{1}$ with $\mathbf{2}$ were carried out under high vacuum at $-40^{\circ} \mathrm{C}$ with phosphorus pentafluoride as initiator in anhydrous methylene chloride. The reactions were terminated by adding methanol into the reaction mixtures, and the copolymers were purified by reprecipitation using chloroform-methanol system. Finally, the copolymers were lyophilized from benzene solutions.

\section{RESULTS AND DISCUSSION}

Copolymerizations of glucosamine monomer (1) with carboxyl-containing monomer (2) were carried out under cationic conditions.

The results of copolymerizations are summarized in Table I. The copolymer composition, i.e., mole fraction of each monomeric unit in the copolymer was calculated by ${ }^{1} \mathrm{H}$ NMR spectroscopy by comparing the intensity of the peak of $\alpha$-methyl protons of 1-(methoxycarbonyl)ethyl group $(1.2 \mathrm{ppm})$ with that of the aromatic protons (7.2 ppm).

As shown in Table I, 1 was efficiently incorporated into polymer chains. As the mole fraction of 1 in the feed increased, polymer yield decreased and the molecular weight of the copolymer decreased. From the copolymerization data, the monomer reactivity ratios at $-40^{\circ} \mathrm{C}$ were evaluated by the Kelen-Tüdös method. ${ }^{14}$ The

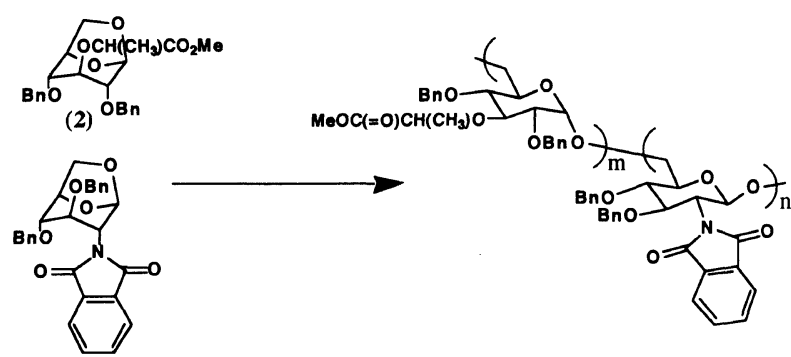

(1) 
Table I. Copolymerization of 1,6-anhydro-3,4-di- $O$-benzyl2-deoxy-2-phthalimido- $\beta$-D-glucopyranose (1) with 1,6 anhydro-2,4-di- $O$-benzyl-3- $O$-1-(methoxycarbonyl)ethyl$\beta$-D-glucopyranose $(2)^{\mathrm{a}}$

\begin{tabular}{|c|c|c|c|c|c|}
\hline \multirow{2}{*}{ No. } & \multirow{2}{*}{$\begin{array}{l}\text { Mole fraction } \\
\text { of } 1 \text { in feed }\end{array}$} & \multirow{2}{*}{$\frac{\text { Time }}{\mathrm{h}}$} & \multirow{2}{*}{$\frac{\text { Yield }}{\%}$} & \multirow{2}{*}{$\begin{array}{l}\text { Mole fraction of } \\
1 \text { in copolymer }\end{array}$} & \multirow{2}{*}{$\begin{array}{c}\bar{M}_{n}^{\mathrm{c}} \\
\left(\times 10^{4}\right)\end{array}$} \\
\hline & & & & & \\
\hline 1 & 0.10 & 0.5 & 56.4 & 0.10 & 8.5 \\
\hline 2 & 0.30 & 3 & 63.1 & 0.31 & 3.8 \\
\hline 3 & 0.50 & 6 & 42.8 & 0.40 & 2.5 \\
\hline 4 & 0.70 & 24 & 32.4 & 0.48 & 1.1 \\
\hline 5 & 0.90 & 24 & 18.6 & 0.63 & 0.7 \\
\hline
\end{tabular}

a Total monomers, $0.70 \mathrm{mmol}$; initiator $\mathrm{PF}_{5}, 10 \mathrm{~mol} \%$ to total monomers; solvent, dichloromethane, $1.5 \mathrm{ml}$; temperature, $-40^{\circ} \mathrm{C}$. ${ }^{b}$ Calculated from ${ }^{1} \mathrm{H}$ NMR data. ${ }^{\mathrm{c}}$ Determined by GPC (polystyrene standard).

monomer reactivity ratios were $r_{1}=0.06 \pm 0.02$ and $r_{2}=0.63 \pm 0.05$. The monomer composition in the feed which gives a certain composition of copolymer can be calculated by the monomer reactivity ratios. For example, mole fraction of 1 of 0.76 in the feed gives $1: 1$ copolymer. The calculated number average sequence length of each monomeric unit in the $1: 1$ copolymer was $1+\left(r_{1} r_{2}\right)^{1 / 2}$. The copolymerizations with different $r_{1} r_{2}$ value provides a certain composition of the copolymer with different sequence length. Therefore, the distribution of each monomeric unit along the copolysaccharide chain may be controlled by the product of the monomer reactivity ratios (kinds of monomers) and monomer composition in the feed. The calculated value of the number average sequence length of each monomeric unit in $1: 1$ copolymer of 1 and 2 is $1.19 \pm 0.04$, indicating that the copolymerization of $\mathbf{1}$ with $\mathbf{2}$ has a tendency to alternate. The copolymer no. 4 in Table I has the number average sequence length of 1 (glucosamine unit) of less than 1.2 and that of $\mathbf{2}$ (carboxyl-containing glucose unit) of less than 1.3.

Homopolymerizability of 1,6 -anhydro-3,4-di- $O$-benzyl-2-deoxy-2-phthalimido- $\beta$-D-glucopyranose (1) is low ${ }^{12}$ and in the copolymerization of 1 with 1,6 -anhydro- 2,3,4-tri- $O$-benzyl- $\beta$-D-glucopyranose (3), the monomer reactivity ratios calculated to be $r_{1}=0.02$ and $r_{3}=1.06$, showing that the reactivity of $\mathbf{1}$ is as high as $\mathbf{3}$ against the highly-active cation derived from 3 , while it is quite low against the propagating chain end cation derived from 1 probably because of the mismatched pair or the steric hindrance. ${ }^{12}$ On the other hand, 1,6-anhydro2,4-di- $O$-benzyl-3- $O$-1-(methoxycarbonyl)ethyl- $\beta$-Dglucopyranose (2) copolymerized with 3 with monomer reactivity ratios of $r_{2}=0.77$ and $r_{3}=0.71$, indicating that each monomer tends to react to an active end of the other one. ${ }^{15}$

Acknowledgments. This work was supported in part by a Grant-in-Aid for Scientific Research (No. 5150) from the Ministry of Education, Science, Sports, and Culture of Japan.

\section{REFERENCES}

1. K. Nagasawa and Y. Inoue, Methods Carbohydr. Chem., 8, 291 (1980).

2. K. Nagasawa and Y. Inoue, Carbohydr. Res., 46, 87 (1976).

3. H. Uchiyama and K. Nagasawa, J. Biol. Chem., 266, 6756 (1991).

4. I. J. Goldstein, G. W. Hay, B. A. Lewis, and F. Smith, Methods Carbohydr. Chem., 5, 361 (1965).

5. K. Nagasawa and Y. Inoue, Carbohydr. Res., 111, 113 (1982).

6. B. Casu, Adv. Carbohydr. Chem. Biochem., 43, 85 (1985).

7. M.-C. Bourin and U. Lindahl, Biochem. J., 289, 313 (1993).

8. C. A. A. van Boeckel and M. Petitou, Angew. Chem. Int. Ed. Engl., 32, 1671 (1993).

9. D. M. Tollefsen, M. E. Peaccocck, and W. J. Monafo, J. Biol. Chem., 261, 8854 (1986).

10. J. N. Liang, B. Chakrabarti, L. Ayotte, and A. S. Perin, Carbohydr. Res., 106, 101 (1982).

11. T. C. Wright, J. J. Castellot, M. Petitou, J.-C. Lormeau, J. Choay, and M. J. Karnovsky, J. Biol. Chem., 264, 1534 (1989).

12. K.-I. Kanno, Y. Kobayashi, S.-I. Nishimura, H. Kuzuhara, and K. Hatanaka, J. Carbohydr. Chem., 14, 481 (1995).

13. K. Hatanaka, T. Uryu, S. Miyahara, and H. Kuzuhara, J. Polym. Sci., A, Polym. Chem., 30, 1223 (1992).

14. T. Kelen and F. Tüdös, J. Macromol. Sci. Chem., A9, 1 (1975).

15. K. Hatanaka, T. Minamisawa, and K.-I. Kanno, Polym. J., 27, 1016 (1995). 